
AS DETERMINANTS OF HEALTH STATUS AND HEALTHCARE UTILISATION IN ELDERLY MEN IN BRAZIL

doi:10.1136/jech.2011.142976p.79

L M Santiago,* I E Mattos, L L Luz. Oswaldo Cruz Foundation, National School of Public Health, Rio de Janeiro, Brazil

Introduction In Latin-America, social inequalities play an important role as determinants of health conditions. This study aimed to analyse associations among social inequalities, health status and healthcare utilisation in Brazilian elderly men.

Methods The study was based on 2859 old men living in a medium size city in Southeast Brazil. Two-stage cluster analysis was performed using socioeconomic and demographic characteristics. To verify possible associations between cluster characteristics and health variables prevalence ratios (PR) and their 95\% CI were estimated.

Results Three clusters were identified. Individuals in Cluster $\mathbf{1}$ (socioeconomically favourable) were married, owned their homes and no longer worked. In Cluster 2 (socioeconomically intermediate) were the younger and still economically active men and in Cluster 3 (socioeconomically unfavourable) were mostly widowers with few years of schooling. In comparison to Cluster 1, men in Cluster 3 had high probability of referring use of public health services (PR 1.17 95\% CI 1.10 to 1.24), report chronic conditions (PR 1.12 95\% CI 1.02 to 1.23) and have no regular physician (PR 1.30 95\% CI 1.17 to 1.44); men in Cluster 2 had low probability of referring poor health (PR $0.6295 \%$ CI 0.52 to 0.63 ), reporting chronic conditions (PR $0.7095 \%$ CI 0.63 to 0.79 ) and relating regular use of medication (PR $0.7795 \%$ CI 0.71 to 0.83 ).

Conclusion Socioeconomic differences are associated with health status and use of health services in this population. Social inequalities leading to poor living conditions and other environmental exposures further enhance their vulnerability. Measures to identify these high risk individuals, reduce inequalities and facilitate the access to health services are necessary in order to reverse current standards.

\section{SP6-9 HEALTH SURVEY IN CAMPINAS (ISACAMP): COMPARISON OF ESTIMATES ACCORDING TO OWNERSHIP OF A RESIDENTIAL TELEPHONE LINE}

doi:10.1136/jech.2011.142976p.80

${ }^{1} \mathrm{P}$ Francisco, ${ }^{*}{ }^{1} \mathrm{M}$ Barros, ${ }^{2} \mathrm{~N}$ Segri, ${ }^{3} \mathrm{C}$ Alves, ${ }^{2} \mathrm{C}$ Cesar. ${ }^{1}$ University of Campinas, Campinas, São Paulo, Brazil; ' University of São Paulo, São Paulo, São Paulo, Brazil; ${ }^{3}$ Institute of Health, São Paulo, São Paulo, Brazil

The aim of this study was to evaluate differences in the sociodemographic and health characteristics among adults (18 years or older) with and without a residential telephone line, using data from the Campinas Health Survey (ISACamp), Brazil, a population-based cross-sectional study carried out in 2008 and 2009. Descriptive statistics and $\chi^{2}$ tests were calculated for these variables. It also estimated the bias associated with non-coverage of the population without telephones before and after the use of post-stratification adjustments. The bias impact on the CIs of these estimates was assessed in terms of accuracy achieved. Approximately $76 \%$ of respondents owned a residential telephone line in Campinas. It was possible to identify differences in socio-demographic data for the population according to the ownership of telephone line, except for marital status. The analysed data also showed that people with telephone had higher prevalence of self-reported osteoporosis, drug use in the 3 days prior to the interview, dental visits in the last 12 months, mammography in women 40 years or more and affiliation to medical plan of health, compared to respondents without a telephone. After the post-stratification adjustment, there were reductions in the bias of estimates for those variables associated with the ownership of telephone line. In this study, except for osteoporosis, post-stratification adjustment was insufficient to correct the bias of non-coverage. Studies with samples where not all units in the target population are likely to be selected as the telephone interview, should consider the potential biases embedded in each variable.

\section{SP6-10 PROCALCITONIN AND WBC, ESR, CRP ALTERATIONS IN CHILDREN WITH SYSTEMIC INFLAMMATORY RESPONSE SYNDROME BEFORE AND AFTER TREATMENT}

doi:10.1136/jech.2011.142976p.81

N Esmailnasab, ${ }^{*}$ A Afkhamzadh, M Abedini. Kurdistan University of medical sciences, Sanandaj/Kurdistan, Iran

Introduction Systemic inflammatory response syndrome (SIRS) due to infection is an important cause of morbidity and mortality in children. The present prospective observational study aimed to determine correlation between Procalcitonin (PCT) and WBC, ESR and $\mathrm{C}$-reactive protein in toddlers before and after treatment.

Methods Overall, 50 patients aged 1-36 months who were hospitalised at the Paediatrics Ward in Besat Hospital in Sanansaj city western Iran through a census sampling method were recruited CBC was measured via cell counter; ESR by Westergren method, CRP via semi quantitative method and PCT via semi quantitative immunochromatography method. Pearson correlation coefficient was used to estimate correlation between WBC, ESR, CRP and PCT before and after treatment of SIRS.

Results Correlation coefficients between PCT and CRP were determined as "Good" and between PCT and ESR was determined as "Mean" in the first day of trial before treatment was started. However, there was no significant correlation between PCT and WBC. In third day of treatment, no significant correlation was found between PCT and WBC count, ESR, CRP.

Conclusions In the onset of treatment and for confirming the diagnosis of SIRS, Procalcitonin and CRP are more favourable values. PCT would be more useful marker to be considered for fallow up procedures.

\section{SP6-11 VALIDITY AND RELIABILITY OF THE WORK ABILITY INDEX QUESTIONNAIRE IN NURSE'S WORK}

doi:10.1136/jech.2011.142976p.82

${ }^{1} \mathrm{~S} H \mathrm{~A}$ da Silva Junior, ${ }^{*} \mathrm{~A} \mathrm{G} \mathrm{G}$ Vasconcelos, ${ }^{2} \mathrm{R} \mathrm{H}$ Griep, ${ }^{2} \mathrm{~L}$ Rotenberg. ${ }^{1} \mathrm{ENSP} /$ FIOCRUZ, Rio de Janeiro, Brazil; ${ }^{2}$ IOC/FIOCRUZ, Rio de Janeiro, Brazil

Introduction The Work Ability Index questionnaire (WAI) assesses the perception of the worker in relation "how well a worker is or will be in the present or near future and how capable this worker is of performing work given the demands of the job and the health status and mental and physical capabilities of the worker." Aims: to investigate the validity and reliability of the WAI in 1436 nurses' work of tree public hospital of the Rio de Janeiro.

Methods We evaluated the validity dimensional through exploratory factor analysis using polychoric correlation matrix and parallel analysis for retention of factors and correlational validity correlating the WAI with theoretically relevant constructs using Spearman correlation. The reliability was assessed by the Cronbach's $\alpha$ and McDonald's $\omega$ coefficients. The factorability of data was tested by the Meyer-Olkin index (KMO) and Bartlett's Test of Sphericity. The $\mathrm{R}$ software was used for analysis. 